
temps de la convergence?

\title{
Efficience et économie d'échelle. Une étude sur l'édition de revues en libre accès
}

Efficiency and Economies of Scale: A Study of the Publishing of Open Access Journals

Joachim Schopfel, Hélène Prost et Jan Erik Frantsvåg

\section{OpenEdition Journals}

\section{Édition électronique}

URL : http://journals.openedition.org/edc/3420

DOI : 10.4000/edc.3420

ISSN : 2101-0366

\section{Éditeur}

Université Lille-3

\section{Édition imprimée}

Date de publication : 30 juin 2012

Pagination : 167-185

ISBN : 978-2-917562-07-9

ISSN : $1270-684$

\section{Référence électronique}

Joachim Schopfel, Hélène Prost et Jan Erik Frantsvåg, « Efficience et économie d'échelle. Une étude sur l'édition de revues en libre accès », Études de communication [En ligne], 38 | 2012, mis en ligne le 30 juin 2014, consulté le 30 avril 2019. URL : http://journals.openedition.org/edc/3420 ; DOI : 10.4000/ edc. 3420

Ce document a été généré automatiquement le 30 avril 2019.

(c) Tous droits réservés 


\title{
Efficience et économie d'échelle. Une étude sur l'édition de revues en libre accès
}

\author{
Efficiency and Economies of Scale: A Study of the Publishing of Open Access \\ Journals
}

Joachim Schopfel, Hélène Prost et Jan Erik Frantsvåg

1 Le passage au numérique du système de publication scientifique peut être considéré comme une incitation en faveur du libre accès, aussi bien du point de vue socio-technique qu'économique (Mounier, 2010). À peine dix ans après la déclaration de Budapest qui propagea l'idée des revues en libre accès ( revues $\mathrm{OA} »)$, le répertoire DOAJ ${ }^{1}$ recense plus de 6500 titres $\mathrm{OA}$, publiés dans 111 pays et couvrant toutes les disciplines. Leur production annuelle a été estimée à 200000 articles, au rythme d'une croissance rapide autour de $20 \%$ par an (Laakso et al., 2011). En moyenne, quatre nouveaux titres OA sont ajoutés chaque jour au DOAJ. Parmi ces titres se trouvent aussi des revues traditionnelles (abonnement payant, modèle du «lecteur-payeur»), bénéficiant parfois d'une forte notoriété, converties au modèle du libre accès (Suber, 2011).

2 Ces publications représentent 6-8\% des articles indexés par les grandes bases de données scientifiques (Suber, 2011). Si l'on prend en compte les articles déposés dans les archives ouvertes, les articles en accès libre correspondent déjà à $20 \%$ de la production scientifique (Björk et al., 2010). Certes, ce taux et l'impact du libre accès varient en fonction de la discipline scientifique (cf. Walters \& Linvill, 2011). Néanmoins, tout laisse à penser que le nombre d'articles publiés dans des revues OA continuera d'augmenter à un rythme soutenu².

Comparées aux revues traditionnelles, ces revues $\mathrm{OA}$ répondent « aux mêmes critères d'exigence et de qualité [...] et (leur) réalisation a un coût » (GFII, 2010). Les différences sont ailleurs. Un des traits distinctifs est l'aspect économique. L'économie, ici, veut dire utiliser au mieux, d'une manière efficiente, les ressources disponibles pour optimiser le résultat escompté. 
4 Pour un éditeur scientifique au sein d'une université ou d'un laboratoire et $a$ fortiori, pour un éditeur de revues $\mathrm{OA}$, l'objectif sera probablement d'augmenter la diffusion et l'impact du contenu scientifique et aussi, de maîtriser les dépenses.

5 Toute utilisation inefficiente des ressources portera préjudice à cet objectif tandis qu'une meilleure efficience économique augmentera la diffusion de contenu au même coût, ou baissera le coût pour une diffusion dans les mêmes proportions. Dans les deux cas, l'efficience sera bénéfique pour la science.

6 Une étude récente a démontré que le secteur $\mathrm{OA}$ est dominé par des éditeurs de petite taille qui souvent ne publient qu'un seul titre (Frantsvåg, 2010). Neuf éditeurs sur dix font partie de cette catégorie, et seulement $25 \%$ des revues OA sont publiées par des éditeurs plus importants. Ce taux est inférieur à l'édition traditionnelle, un fait qui n'est pas sans conséquence sur l'économie de la publication.

7 Quel est l'intérêt d'étudier la taille d'un éditeur, selon le nombre de revues qu'il publie? Pour comprendre ce lien entre la taille de l'éditeur et l'efficience, il faut introduire deux autres concepts, les coûts fixes et l'économie d'échelle.

8 Les coûts fixes sont les dépenses liées à la production ou à la prestation de service ; cellesci sont indépendantes du volume ou nombre des biens, produits ou services. Dans notre cas, il s'agit de dépenses qui ne sont pas liées au nombre d'articles ou de revues publiés. Pour l'édition de revues OA, les coûts fixes sont par exemple les frais de location de matériels ou locaux, frais de maintenance et d'infrastructure (serveur), etc. De même, l'amortissement d'un investissement, les frais d'une formation, etc. font partie des coûts fixes. La somme de ces dépenses annuelles, divisée par le nombre d'articles ou de revues publiés, représente le coût fixe moyen ou unitaire d'un article ou d'une revue.

9 L'économie d'échelle correspond à la baisse du coût unitaire (ou coût moyen) qu'on obtient en augmentant la production. Un nombre plus élevé de revues baisse le coût fixe moyen d'un titre mais favorise aussi une plus grande compétence et expérience du personnel. Du fait d'une meilleure maîtrise des procédures et des outils, cela améliore également l'efficacité technique et organisationnelle (spécialisation). Mais la taille d'un éditeur peut avoir d'autres effets, sur le degré de financement et sur la visibilité par exemple.

10 Financement: Les premiers résultats du projet $\mathrm{SOAP}^{3}$ révèlent des différences importantes du financement entre grands et petits éditeurs $\mathrm{OA}$, ces derniers dépendant beaucoup plus des subventions publiques (Polydoratou et al., 2010).

11 Visibilité : Sur le site DOAJ, moins de la moitié des revues OA peuvent être interrogées au niveau des articles. Fournir les métadonnées des articles augmente la diffusion du contenu. Disposant de davantage de moyens et personnels, les grands éditeurs profitent généralement de cette fonctionnalité gratuite et s'assurent que leurs métadonnées soient accessibles via le DOAJ. Les petits éditeurs par contre l'utilisent peu parce qu'ils n'ont pas le bon logiciel, parce qu'ils n'ont pas les moyens ou compétences, ou simplement parce qu'ils n'en saisissent pas l'intérêt. Dans tous les cas de figure, le résultat est le même : une diffusion moins efficiente du contenu, comparé aux grands éditeurs.

12 Archivage pérenne: la taille d'un éditeur $\mathrm{OA}$ a également des répercussions sur la possibilité d'accès aux services d'archivage pérenne tels que Portico ou LOCKSS/CLOCKSS (Seadle, 2011). 
13 Walters \& Linvill (2011) supposent un lien entre la viabilité (longévité) d'une revue et le nombre d'articles publiés. D'une manière plus générale, l'importance et la notoriété du catalogue des titres donnent un avantage économique à l'éditeur qui peut jouer sur un « effet de marque » pour définir sa stratégie commerciale (Vajou et al., 2009).

L'article réunit un certain nombre d'éléments sur la distribution des éditeurs de revues en libre accès en fonction de leur taille afin d'évaluer l'impact de cette distribution sur l'édition scientifique. L'article a trois objectifs : consolider l'étude de Frantsvåg (2010) sur la distribution des éditeurs de revues OA avec des données de 2011 ; identifier l'évolution de la situation depuis 2010 ; et étudier plus particulièrement le marché des revues OA en France. Il contribue ainsi à l'analyse et à la compréhension économique de l'édition scientifique en général et du libre accès en particulier; l'article décrit en parallèle d'autres approches comme l'analyse des coûts de production, des subventions, du retour sur investissement, de la structure de l'offre du marché, etc. ${ }^{4}$.

\section{Méthodologie}

Pour constituer la liste des éditeurs, nous avons suivi la méthodologie décrite par Frantsvåg (2010). Nous avons extrait les données sur les revues et éditeurs du répertoire des revues en libre accès DOAJ ${ }^{5}$. De ce fichier nous avons retiré les titres morts et suspendus pour ne garder que des titres vivants $(\mathrm{N}=6446)$. A chaque revue $\mathrm{OA}$ est associé un éditeur. Après avoir corrigé plusieurs erreurs ou variantes d'écriture, nous avons obtenu un fichier avec 3921 éditeurs. À chaque éditeur sont associées entre une et plus de 200 revues en libre accès (OA). Ce fichier a servi de base pour l'analyse et le classement des éditeurs $\mathrm{OA}$ en fonction de leur taille.

Dans un deuxième temps, nous avons comparé les éditeurs de revues en libre accès (OA) avec les éditeurs de revues payantes (toll access ou TA). Comme le DOAJ ne contient pas de revues payantes, nous avons eu recours à la base Ulrichsweb de ProQuest ${ }^{6}$, dont nous avons obtenu une extraction ${ }^{7}$ de toutes les revues scientifiques ${ }^{8}$. Ce fichier contient 23734 titres publiés par 8102 éditeurs et permet de différencier entre les éditeurs OA, les éditeurs traditionnels de revues payantes (TA) et les éditeurs mixtes avec des revues $\mathrm{OA}$ et TA. Ce deuxième fichier a été analysé de la même façon que le premier, en comparant les résultats par type d'éditeur.

17 Pour finir, nous avons opéré un zoom sur l'édition scientifique en France, avec la même méthodologie. Dans les deux fichiers DOAJ et Ulrichsweb, nous avons ainsi sélectionné les revues publiées par un éditeur français, indépendamment de leur langue de publication (française ou autre). Sur ces deux fichiers, nous avons effectué les mêmes analyses statistiques (classement par taille des éditeurs, regroupement, comptage des revues) et nous avons comparé les résultats avec l'échantillon initial.

L'originalité de cette approche est double : le plus souvent, les études sur les revues en libre accès se limitent à une analyse de leurs caractéristiques telles que le pays d'édition, la langue, ou la discipline. La comparaison avec les revues traditionnelles est assez rare, et l'analyse à partir des éditeurs et de leur taille est l'exception. La deuxième particularité est liée à une méthodologie assez robuste offrant des résultats suivis d'une analyse plus fine et/ou qualitative. 


\section{Résultats}

\section{Éditeurs de revues $O A$}

Le répertoire DOAJ contient 3921 éditeurs de revues en libre accès. La plus grande majorité de ces éditeurs ne publie qu'un seul titre (tableau 1 ).

Tableau 1 : Nombre d'éditeurs et de revues OA

\begin{tabular}{|c|c|c|c|c|}
\hline \multirow{2}{*}{$\begin{array}{l}2011 \\
\text { Taille éditeur }\end{array}$} & \multicolumn{2}{|l|}{ Nombre } & \multicolumn{2}{|c|}{ En $\%$ du total } \\
\hline & Éditeurs & Revues & Éditeurs & Revues \\
\hline 1 & 3397 & 3397 & $86,6 \%$ & $52,7 \%$ \\
\hline 2 & 280 & 560 & $7,1 \%$ & $8,7 \%$ \\
\hline 3 & 79 & 237 & $2,0 \%$ & $3,7 \%$ \\
\hline 4 & 43 & 172 & $1,1 \%$ & $2,7 \%$ \\
\hline 5 & 25 & 125 & $0,6 \%$ & $1,9 \%$ \\
\hline 6 & 15 & 90 & $0,4 \%$ & $1,4 \%$ \\
\hline 7 & 11 & 77 & $0,3 \%$ & $1,2 \%$ \\
\hline 8 & 8 & 64 & $0,2 \%$ & $1,0 \%$ \\
\hline 9 & 9 & 81 & $0,2 \%$ & $1,3 \%$ \\
\hline 10 & 6 & 60 & $0,2 \%$ & $0,9 \%$ \\
\hline $11-20$ & 32 & 434 & $0,8 \%$ & $6,7 \%$ \\
\hline $21-50$ & 9 & 251 & $0,2 \%$ & $3,9 \%$ \\
\hline $51-100$ & 4 & 267 & $0,1 \%$ & $4,1 \%$ \\
\hline $100+$ & 3 & 631 & $0,1 \%$ & $9,8 \%$ \\
\hline Total & 3921 & 6446 & $100 \%$ & $100 \%$ \\
\hline
\end{tabular}

(Source : DOAJ juin 2011)

$95,8 \%$ des éditeurs publient jusqu'à trois titres; et $65,1 \%$ titres sont édités par ces éditeurs de petite ou très petite taille.

21 De l'autre côté de l'échelle se trouvent 54 éditeurs $(1,4 \%)$ qui éditent 10 titres ou plus, cumulant ainsi 1643 revues, ce qui représente $25,5 \%$ du total des titres OA. En tête du classement, on trouve les trois éditeurs BioMed Central, Hindawi et Bentham Open, 
proposant plus de 200 revues OA chacun, suivis par quatre éditeurs spécialisés en sciences médicales (Medknow Publications, Dove Medical Press, ISPUB Internet Scientific Publications, Libertas Academica).

Pour $44 \%$ des revues OA, le DOAJ propose un accès aux articles via les métadonnées, augmentant ainsi leur visibilité. $22 \%$ des revues indiquent une diffusion sous une licence Creative Commons.

\section{Comparaison avec d'autres éditeurs}

La même démarche appliquée aux éditeurs et revues du modèle traditionnel (TA) produit un autre résultat (tableau 2).

Tableau 2 : Nombre d'éditeurs et de revues TA

\begin{tabular}{|c|c|c|c|c|}
\hline \multirow{2}{*}{$\begin{array}{l}2011 \\
\text { Taille éditeur }\end{array}$} & \multicolumn{2}{|l|}{ Nombre } & \multicolumn{2}{|c|}{ En \% du total } \\
\hline & Éditeurs & Revues & Éditeurs & Revues \\
\hline 1 & 6671 & 6671 & $82,3 \%$ & $28,1 \%$ \\
\hline 2 & 646 & 1292 & $8,0 \%$ & $5,4 \%$ \\
\hline 3 & 200 & 600 & $2,5 \%$ & $2,5 \%$ \\
\hline 4 & 121 & 484 & $1,5 \%$ & $2,0 \%$ \\
\hline 5 & 76 & 380 & $0,9 \%$ & $1,6 \%$ \\
\hline 6 & 55 & 330 & $0,7 \%$ & $1,4 \%$ \\
\hline 7 & 31 & 217 & $0,4 \%$ & $0,9 \%$ \\
\hline 8 & 29 & 232 & $0,4 \%$ & $1,0 \%$ \\
\hline 9 & 27 & 243 & $0,3 \%$ & $1,0 \%$ \\
\hline 10 & 22 & 220 & $0,3 \%$ & $0,9 \%$ \\
\hline $11-20$ & 92 & 1326 & $1,1 \%$ & $5,6 \%$ \\
\hline $21-50$ & 75 & 2295 & $0,9 \%$ & $9,7 \%$ \\
\hline $51-100$ & 29 & 2159 & $0,4 \%$ & $9,1 \%$ \\
\hline $100+$ & 28 & 7285 & $0,3 \%$ & $30,7 \%$ \\
\hline Total & 8102 & 23734 & $100 \%$ & $100 \%$ \\
\hline
\end{tabular}

(Source : Ulrichsweb juin 2011) 
Certes, le nombre d'éditeurs publiant un, deux ou trois titres est également élevé, avec $92,8 \%$ de la liste des éditeurs ; mais leur catalogue ne correspond qu'à $36,1 \%$ des titres. La différence entre éditeurs de revues OA et TA est dans la distribution globale et surtout, en fin de classement. 246 éditeurs ( $3 \%$ ) publient dix titres ou plus, ce qui représente un total de 13285 revues (56\%). On trouve ici les grands noms de l'édition commerciale (Elsevier, Springer, Wiley-Blackwell, Taylor \& Francis, etc.); la concentration est donc bien plus élevée.

\section{Évolution depuis 2010}

Comment ces chiffres ont-ils évolués depuis 2010? Le répertoire DOAJ indique une augmentation de 690 éditeurs (+ $21 \%$ ) et de 1285 revues OA (+ $25 \%)$ en un an (tableau 3).

À titre de comparaison, selon les chiffres d'Ulrichsweb, le nombre d'éditeurs traditionnels a légèrement baissé $(-5,4 \%)$ tandis que le nombre de revues TA a augmenté au rythme de $4,9 \%$ ce qui correspond assez bien à la modélisation de Mabe (2003) selon laquelle le taux de croissance des revues oscille sur le long terme entre $3 \%$ et $4,5 \%$.

Mais plus intéressante est la comparaison des chiffres absolus : depuis 2010, il y a eu davantage de nouveaux titres OA que de nouveaux titres TA (+ $15 \%)$.

Tableau 3 : Évolution du nombre d'éditeurs et de revues OA

\begin{tabular}{|c|c|c|c|c|}
\hline \multirow{2}{*}{$\begin{array}{l}\text { 2010/2011 } \\
\text { Taille éditeur }\end{array}$} & \multicolumn{2}{|c|}{ Évolution en nb } & \multicolumn{2}{|c|}{ Évolution en \% } \\
\hline & Éditeurs & Revues & Éditeurs & Revues \\
\hline 1 & 558 & 558 & $17,3 \%$ & $10,8 \%$ \\
\hline 2 & 68 & 136 & $2,1 \%$ & $2,6 \%$ \\
\hline 3 & 29 & 87 & $0,9 \%$ & $1,7 \%$ \\
\hline 4 & 13 & 52 & $0,4 \%$ & $1,0 \%$ \\
\hline 5 & 9 & 45 & $0,3 \%$ & $0,9 \%$ \\
\hline 6 & -1 & -6 & $0,0 \%$ & $-0,1 \%$ \\
\hline 7 & -2 & -14 & $-0,1 \%$ & $-0,3 \%$ \\
\hline 8 & -1 & -8 & $0,0 \%$ & $-0,2 \%$ \\
\hline 9 & 2 & 18 & $0,1 \%$ & $0,3 \%$ \\
\hline 10 & 4 & 40 & $0,1 \%$ & $0,8 \%$ \\
\hline $11-20$ & 6 & 77 & $0,2 \%$ & $1,5 \%$ \\
\hline $21-50$ & 3 & 68 & $0,1 \%$ & $1,3 \%$ \\
\hline $51-100$ & 2 & 135 & $0,1 \%$ & $2,6 \%$ \\
\hline
\end{tabular}




\begin{tabular}{|l|l|l|l|l|}
\hline $100+$ & 0 & 97 & $0,0 \%$ & $1,9 \%$ \\
\hline Total & 690 & 1285 & $21,4 \%$ & $24,9 \%$ \\
\hline
\end{tabular}

(Source : DOAJ juin 2011)

Tableau 4 : Nombre d'éditeurs et de revues OA en France

\begin{tabular}{|l|l|l|l|l|}
\hline 2011 & \multicolumn{2}{|l|}{ Nombre } & \multicolumn{2}{l|}{ En \% du total } \\
\hline Taille éditeur & Éditeurs & Revues & Éditeurs & Revues \\
\hline 1 & 111 & 111 & $93,3 \%$ & $83,5 \%$ \\
\hline 2 & 5 & 10 & $4,2 \%$ & $7,5 \%$ \\
\hline 3 & 2 & 6 & $1,7 \%$ & $4,5 \%$ \\
\hline 6 & 1 & 6 & $0,8 \%$ & $4,5 \%$ \\
\hline Total & 119 & 133 & $100 \%$ & $100 \%$ \\
\hline
\end{tabular}

(Source : DOAJ juin 2011)

\section{La situation en France}

On peut également constater une légère concentration dans la mesure où le nombre de revues éditées par des éditeurs gérant plus de dix titres a augmenté de $8 \%$. Parmi les éditeurs proposant plus de cinquante titres apparaissent deux nouveaux éditeurs ; ce sont Libertas Academica et Dove Medical Press. Mais d'une manière générale, l'édition des revues en libre accès reste encore pour l'instant l'affaire de petits éditeurs.

Le DOAJ répertorie 133 revues françaises en libre accès. Voici la répartition (tableau 4) :

D'après le DOAJ, 83,5\% des revues OA en France sont des « titres uniques ", publiés par un laboratoire, une école, un établissement. Deux organismes publient trois revues en OA (Muséum National d'Histoire Naturelle, Sorbonne). L'unique éditeur français qui développe un réel catalogue de revues OA est EDP Sciences ${ }^{9}$ avec six titres répertoriés dans le DOAJ, dont les Annales de Toxicologie Analytique, les Europhysics News et le Journal sur l'enseignement des sciences et technologies de l'information et des systèmes (J3eA).

31 De ces titres OA, $19 \%$ ont fourni les métadonnées d'articles au DOAJ, et $8 \%$ indiquent une politique de diffusion sous Creative Commons.

32 À titre de comparaison, voici l'information sur l'édition traditionnelle (revues TA) en France, à partir de la base Ulrichsweb. En juin 2011, Ulrichsweb signale 351 revues éditées en France et dotées d'un comité de lecture (tableau 5). 
Tableau 5 : Nombre d'éditeurs et de revues TA en France

\begin{tabular}{|c|c|c|c|c|}
\hline \multirow{2}{*}{$\begin{array}{l}2011 \\
\text { Taille éditeur }\end{array}$} & \multicolumn{2}{|l|}{ Nombre } & \multicolumn{2}{|c|}{ En \% du total } \\
\hline & Éditeurs & Revues & Éditeurs & Revues \\
\hline 1 & 134 & 134 & $84,3 \%$ & $38,2 \%$ \\
\hline 2 & 10 & 20 & $6,3 \%$ & $5,7 \%$ \\
\hline 3 & 2 & 6 & $1,3 \%$ & $1,7 \%$ \\
\hline 4 & 4 & 16 & $2,5 \%$ & $4,6 \%$ \\
\hline 5 & 2 & 10 & $1,3 \%$ & $2,8 \%$ \\
\hline 6 & 2 & 12 & $1,3 \%$ & $3,4 \%$ \\
\hline 7 & 0 & 0 & $0,0 \%$ & $0,0 \%$ \\
\hline 8 & 0 & 0 & $0,0 \%$ & $0,0 \%$ \\
\hline 9 & 0 & 0 & $0,0 \%$ & $0,0 \%$ \\
\hline 10 & 0 & 0 & $0,0 \%$ & $0,0 \%$ \\
\hline $11-20$ & 2 & 24 & $1,3 \%$ & $6,8 \%$ \\
\hline $21-50$ & 2 & 45 & $1,3 \%$ & $12,8 \%$ \\
\hline $51-100$ & 1 & 84 & $0,6 \%$ & $23,9 \%$ \\
\hline \multirow[t]{2}{*}{$100+$} & 0 & 0 & $0,0 \%$ & $0,0 \%$ \\
\hline & 159 & 351 & $100,0 \%$ & $100,0 \%$ \\
\hline
\end{tabular}

(Source : Ulrichsweb juin 2011)

Les chiffres confirment une situation connue (Minon \& Chartron, 2005) : beaucoup de petits éditeurs (surtout en SHS) et peu d'éditeurs d'une taille plus importante ${ }^{10}$ dont un seul à approcher les 100 titres (Elsevier Masson).

\section{Discussion}

La méthodologie appliquée produit des résultats assez fiables mais a quelques limites. Il s'agit surtout de deux sources d'erreurs potentielles: l'identification des éditeurs dans DOAJ et Ulrichsweb (erreurs d'écriture, variantes, etc.) ${ }^{11}$, et la sélection des revues $\mathrm{OA}$ (recensement non exhaustif et hétérogène à partir d'un signalement volontaire, comptage différent entre DOAJ, Ulrichsweb et d'autres outils tels que la EZB ${ }^{12}$ ou le Open Science Directory $\left.{ }^{13}\right)$. En plus, il y a parfois une incertitude sur la notion d'éditeur, le DOAJ privilégie l'éditeur scientifique à l'éditeur commercial (au sens de 
diffusion) ; CLEO (revues.org) par exemple ne figure pas parmi les éditeurs (cf. plus loin). Cette situation pose un problème à toutes les études sur le développement et l'économie des revues OA (Laakso et al., 2011).

L'effet combiné de ces sources d'erreur tend à sous-estimer l'importance de l'offre éditoriale dans le domaine du libre accès. Il est évident par exemple que le nombre de revues en libre accès édités en France est supérieur au recensement par le DOAJ. Il suffit de comparer le nombre de revues SHS sur la plate-forme Revues.org du CLEO ${ }^{14}$ pour s'en rendre compte. Pour autant, ce biais connu et partagé par toutes les études dans ce domaine ne devrait pas influencer les résultats d'analyse d'une manière significative :

- Les éditeurs et revues OA sont moins nombreux que ceux du modèle traditionnel (TA) mais leur nombre augmente plus rapidement.

- La grande majorité des éditeurs $\mathrm{OA}$ sont de petite et de très petite taille (entre un et trois titres).

- Par rapport à l'édition traditionnelle (TA), l'édition OA est bien moins concentrée ; il y a moins de grands éditeurs et moins de revues publiées par de grands éditeurs.

La croissance est certainement liée au potentiel d'auto-production et d'auto-diffusion des revues sur le web (Chartron \& Rebillard, 2004). Dans cette situation d'émiettement, on peut douter de l'efficience de l'édition en libre accès. La part des revues « uniques » en libre accès est bien plus élevée que celle des autres; par contre il y a significativement moins de revues OA éditées par des éditeurs dont le catalogue est important (figure 1).

Figure 1 : Comparaison revues OA et TA (\%)

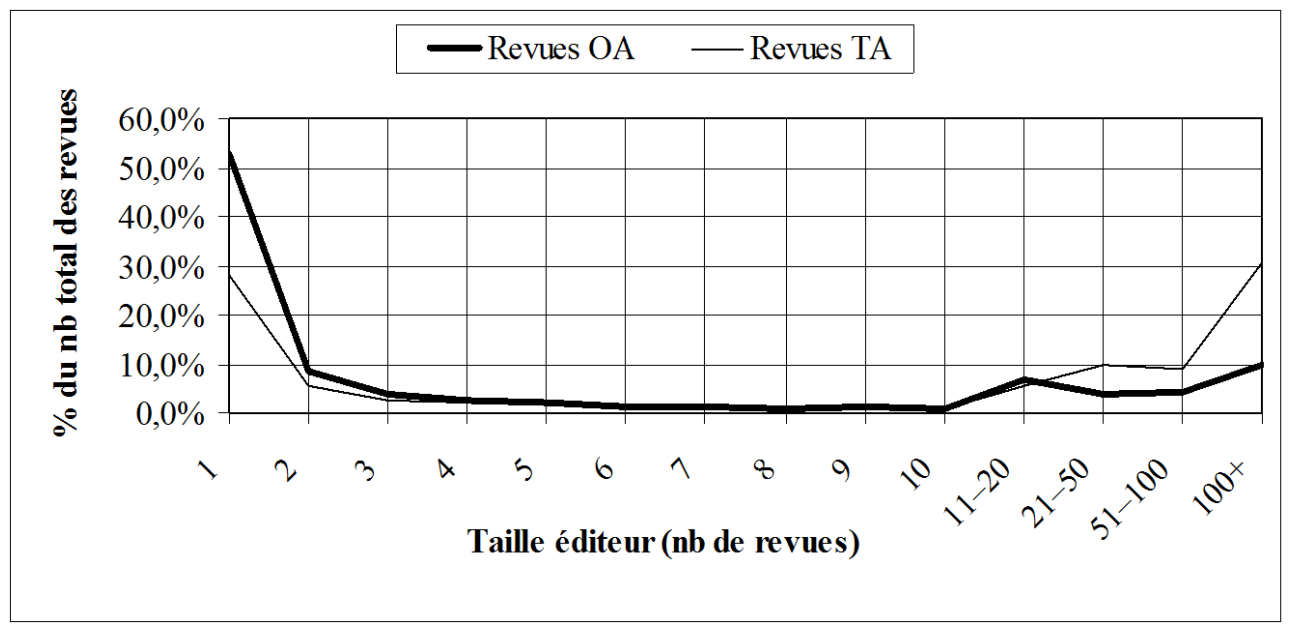

(source : DOAJ et Ulrichsweb juin 2011)

Cette situation « fragmentée » désavantage l'édition OA du point de vue économique, avec une conséquence directe sur la visibilité et l'impact des contenus. Cependant, ce constat invite à plusieurs réflexions.

(1) Premièrement se pose la question de la notion même de l'éditeur, tel qu'il est répertorié par le DOAJ ou par Ulrichsweb. S'agit-il de l'institution et si oui, à quel niveau l'université, le laboratoire, un service de presses universitaires? Ou s'agit-il d'une rédaction ou d'une équipe éditoriale? S'il s'agit d'une institution, les équipements, 
procédures et ressources sont-ils mutualisés entre plusieurs produits et si oui, comment ? Quid de l'éditeur au sens de la diffusion?

Pour illustrer la grande diversité des situations, voici quelques exemples (non corrigés) d'éditeurs référencés par le DOAJ : l'université de Rouen, le laboratoire d'ethnologie et de sociologie comparative (CNRS et l'université Paris 10), les Presses Universitaires de Paris Ouest Nanterre La Défense, l'Association Française de Linguistique Cognitive, le Centre Flaubert, le Centre d'Histoire de Sciences Po, IRIS-EHESS, la Chaire IUF Dynamique du Langage et Contact des Langues, le CEMAGREF, l'École des Hautes Études en Sciences Sociales, etc. L'université côtoie le laboratoire, l'établissement de recherche les presses universitaires, et l'école est citée au même rang qu'une de ses composantes.

Là où plusieurs équipes scientifiques publient des revues $\mathrm{OA}$ sur le même campus, il pourrait être intéressant d'envisager une mutualisation des procédures afin de réaliser une économie d'échelle (Frantsvåg, 2011a).

41 (2) La deuxième réflexion porte sur le lien entre le nombre de revues et l'économie d'échelle. Concrètement, à partir de quel nombre peut-on parler d'une économie d'échelle? Du fait des coûts fixes, l'économie d'échelle n'est certainement pas une fonction linéaire du nombre de revues publiées. Mais existe-t-il une sorte d'effet de seuil ? Si oui, où situer ce seuil - à trois, cinq, dix ou vingt titres? D'autre part, il faudrait également tenir compte du nombre d'articles, avec des cas de figure assez variés. Souvent, les revues OA sont de petit volume, avec peu d'articles. Mais en même temps se développent des «méga-revues » avec des centaines voire des milliers d'articles, comme par exemple PLoS One de la Public Library of Science ou les Scientific Reports du Nature Publishing Group (Frantsvåg, 2011b). Dans les deux cas, l'économie d'échelle est certainement assez différente.

(3) Notre troisième remarque concerne tout particulièrement l'édition des revues $\mathrm{OA}$ en France. L'absence de grands éditeurs STM et le morcellement du paysage de l'édition SHS trouvent leurs reflets dans les caractéristiques de l'édition en libre accès. Que ce soit pour l'ensemble du DOAJ ou pour la France en particulier, le paysage du libre accès est constitué d'une mosaïque d'éditeurs de petite taille dont l'essentiel de la production appartient au domaine des SHS. Au niveau international, $52 \%$ des éditeurs sont du domaine SHS et parmi ces éditeurs, $92 \%$ proposent trois titres au plus et représentent $73 \%$ de la production SHS. La situation est encore plus morcelée du côté des éditeurs français : $75 \%$ des éditeurs relèvent des SHS et parmi eux, $97 \%$ sont des petits éditeurs ( $\leq 3$ titres) qui, ensemble, éditent $93 \%$ de la production SHS.

43 Ceci étant, une partie de ces éditeurs, en SHS mais aussi dans d'autres disciplines, a trouvé le moyen de garder l'autonomie scientifique et commerciale tout en mutualisant une partie des coûts pour la mise en ligne. La solution s'appelle CLEO (revues.org), CAIRN ou INIST (I-Revues). Autrement dit, en adoptant le modèle de mise en ligne sur une plateforme mutualisée, sur laquelle sont éditées plusieurs dizaines ou, dans le cas du CLEO, plusieurs centaines d'autres revues, l'économie d'échelle est réalisée du moins pour une partie du workflow (hébergement, mise en ligne, maintenance, assistance etc.).

L'importance de cette économie dépend de plusieurs facteurs, dont le modèle de production (délégation ou appropriation?) puis le modèle économique (statut privé dans le cas de CAIRN, statut public pour le CLEO et l'INIST). Dans tous les cas de figure, l'éditeur universitaire qui édite un ou deux titres peut tout à fait réaliser une économie d'échelle et publier d'une manière plus efficiente sans augmenter le nombre de produits, en confiant 
la mise en ligne à un établissement qui prend en charge une partie de l'aval de la production, y compris la formation du personnel, la maintenance du système et la conservation des fichiers ${ }^{15}$. Ceci peut aboutir à des modèles complexes, mettant en jeu plusieurs acteurs, dont les statuts et les objectifs sont différents.

(4) Par ailleurs, le concept de la revue OA présente de multiples facettes. Notre étude se contente de comptabiliser les revues en libre accès du DOAJ comme s'il s'agissait du même produit. Or, ce n'est pas le cas. Báscones Dominguez (2006) par exemple distingue quatre types de revues $\mathrm{OA}$, dont des revues partiellement libres et des revues en libre accès uniquement pour des pays en voie de développement, avec néanmoins plusieurs caractéristiques communes: la présence d'un comité de lecture, une structure comparable à celle des revues traditionnelles, pas de version papier (ou seulement moyennant finance), mise en ligne sur une plateforme Web (éditeur ou agrégateur). Sur ce plan aussi, notre étude n'est qu'un début car elle ne fait pas cette distinction et elle exclut la catégorie des revues OA pour les pays en voie de développement et les revues partiellement $\mathrm{OA}^{16}$.

46 (5) Une dernière remarque: L'analyse fait plus ou moins abstraction des disciplines scientifiques. Or, la part des publications en libre accès varie par discipline, allant de $13 \%$ pour la chimie à $33 \%$ en sciences de la terre ${ }^{17}$ (Björk et al., 2010) ; même constat pour le nombre moyen d'articles publiés qui reflète les modes de production et qui par conséquent varie largement selon la discipline scientifique (Walters \& Linvill, 2011).

Existe-t-il pour autant un lien entre discipline et efficience économique ? Nos statistiques indiquent quelques différences. La part des "grands" éditeurs OA (avec au moins 10 titres OA) est plus importante dans les sciences de la vie et parmi les éditeurs multidisciplinaires, tandis que la plus grande concentration du nombre de revues est en médecine. Une analyse plus fine devrait apporter d'autres éléments.

\section{Conclusion}

48 L'analyse de la taille des éditeurs de revues OA pose la question de l'efficience économique de ce modèle de production éditoriale. La situation actuelle caractérisée par un grand nombre de petits éditeurs, ne proposant souvent qu'un seul titre, semble de ce point de vue loin d'être optimale. Or, en ce qui concerne la France, l'étude réalisée par le GFII en 2009 avait déjà identifié le maintien d'un " tissu éditorial SHS », tant public que privé, économiquement viable, comme l'un des enjeux des années à venir. Face à la fragilité économique du secteur, le GFII préconise la mutualisation d'outils et de ressources et la collaboration à toutes les étapes de la chaîne éditoriale.

49 Appliqué à la production des revues $\mathrm{OA}$, cela signifie: la (re)consolidation passera nécessairement par la recherche d'une plus grande efficience et ceci aussi, par la concentration et le regroupement des lieux de production.

Ceci est a priori indépendant de leur modèle économique. Qu'il s'agisse d'un modèle sponsorisé (comme celui du CLEO), d'un modèle du paiement par l'auteur (BioMed Central) ou hybride (SpringerOpen), dans tous les cas de figure le coût de la fabrication sera à prendre en charge par les organismes de l'enseignement supérieur et de la recherche, directement ou indirectement voire les deux.

51 Mais le modèle éditorial du libre accès est-il satisfaisant ou avantageux d'un point de vue économique? La réponse n'est pas simple. Une université par exemple aura tendance à 
confondre coûts et dépenses directes sans tenir compte de l'utilisation des ressources, en particulier le temps de travail des enseignants-chercheurs ${ }^{18}$. De plus, comme le soulignent Walters \& Linvill (2011), les dépenses directes relativement basses du small-scale online publishing peuvent inciter à créer des revues spécialisées sans modèle économique viable ou durable ${ }^{19}$. Mais rares sont encore les études qui analysent l'ensemble des coûts réels d'une publication $\mathrm{OA}^{20}$.

Pour un grand organisme de recherche, tel que le CERN, les frais de publication dépasseraient largement les frais d'abonnement (Báscones Dominguez, 2006). Si les frais passent de ceux qui lisent à ceux qui publient, les organismes qui publient beaucoup payent en général davantage. Si un tel organisme devait payer pour être publié ${ }^{11}$ et tant que ce prix à payer reste élevé, l'intérêt économique d'un modèle $\mathrm{OA}$ resterait limité. Mais si, grâce à une meilleure efficience, les coûts de production baissaient ? Cette meilleure efficience passerait-elle par une mutualisation partielle ou totale de la chaîne éditoriale et/ou par une plus grande concentration de l'édition en libre accès?

Du point de vue technique, il est facile aujourd'hui de créer une revue OA en ligne. Mais une telle entreprise a un coût. Et si en plus, comme c'est souvent le cas, la publication s'adosse à une initiative personnelle, un projet ou une équipe de recherche, un laboratoire dont la durée de vie est limitée par définition, quel modèle protègera cette revue si le contexte changeait?

Pour des raisons économiques il est probable que la concentration de l'édition OA se poursuivra, même si elle n'atteindra peut-être jamais le niveau de l'édition $\mathrm{TA}^{22}$. La différence entre les deux modèles est flagrante pour les grands « catalogues» de plus de cent titres: vingt-huit éditeurs publient ensemble 7285 titres suivant le modèle traditionnel d'abonnement (paiement par lecteur, TA) tandis que dans la même catégorie (>100 titres) trois éditeurs seulement publient 631 titres en libre accès (OA) (tableaux 1 et 2 plus haut).

55 Or, il ne faut pas confondre l'édition en libre accès avec l'édition non-commerciale. En fait, plus d'un quart des revues du DOAJ est (déjà) publié par des éditeurs commerciaux (Walters \& Linvill, 2011) dont on connaît la tendance à la concentration et l'appétit du chiffre d'affaires. Pourquoi ces éditeurs poursuivraient-ils une autre stratégie pour cette catégorie de revues? Une raison de plus de penser qu'à terme, la courbe de l'édition OA se rapprochera davantage de celle de l'édition TA, du moins pour les grands éditeurs et la concentration des catalogues (figure 1 plus haut).

D'autres facteurs favoriseront ce processus de concentration, même si, comme en France, cette concentration ne concernera peut-être pas en premier lieu l'édition mais l'hébergement, la mise en ligne et l'archivage pérenne (CLEO, PERSEE, etc.). Citons au moins deux de ces facteurs :

1. Le besoin de faire des économies non pas pour assurer la production courante mais pour pouvoir investir dans le développement technologique de la publication du futur (services à valeur ajoutée, intégration des données scientifiques, etc.).

2. Les contraintes budgétaires liées à la politique publique en matière d'enseignement supérieur et de la recherche qui se traduit en France par la révision générale des politiques publiques ou encore par l'élargissement des responsabilités et compétences des universités. Elles ne remettront pas nécessairement en question le rôle éditorial des universités et organismes de recherche mais elles poseront certainement un jour ou l'autre la question du financement, des coûts réels et du contrôle des dépenses. Les options seront toujours les 
mêmes : une meilleure gestion, une mutualisation volontariste, une concentration de la production, l'externalisation, enfin la privatisation.

57 Il est certain que notre étude n'est qu'un début et que ses résultats nécessitent une analyse plus approfondie, détaillée et qualitative qui tient compte de la spécificité des champs disciplinaires.

Cependant, vu ce contexte et vu l'évolution du marché de l'édition ${ }^{23}$, on peut déjà se demander si finalement l'avenir de l'édition en libre accès ne sera pas tout simplement l'édition commerciale, au moins pour les services à forte valeur ajoutée ${ }^{24}$. Mais dans ce cas, quid de l'idée de l'information scientifique comme bien commun?

La question est posée. Le problème est économique. Mais la réponse sera politique.

\section{BIBLIOGRAPHIE}

Báscones Dominguez M., (2006), Economics of open access publishing, in Serials : The Journal for the Serials Community, vol. 19, n 1, pp. 52-60.

Bester E., (2010), Les services pour les archives ouvertes : de la référence à l'expertise, in Documentaliste - Sciences de l'Information, vol. 47, $\mathrm{n}^{\circ}$ 4, pp. 4-15.

Björk B.-C. et al., (2010), Open Access to the Scientific Journal Literature: Situation 2009, in PLoS ONE, vol. $5, \mathrm{n}^{\circ} 6$, p. e11273+.

Chartron G., (2010), Scénarios prospectifs pour l'édition scientifique, in Hermès, vol. 57, pp. 123-129.

Chartron G. et Rebillard F., (2004), Modèles de publication sur le web, Rapport d'activités AS-CNRS 103 , rapport, Paris, CNRS-STIC, $63 \mathrm{p}$.

Dewatripont M. et al., (2006), Study on the economic and technical evolution of the scientific information markets in Europe, rapport, Bruxelles, DG Research European Commission, 112 p.

Duvinage E., (2011), Évolution du modèle économique d'Études de Communication dans le contexte du passage au numérique des revues en sciences humaines et sociales, mémoire de Master, Université de Lille 3, 66 p.

Frantsvåg J. E., (2010), The size distribution of open access publishers: A problem for open access?, in First Monday, vol. 15, n 12.

Frantsvåg J. E., (2011a), Would not bigger be better?: some reflections on aspects of the business models of Open Access publishing, mémoire de Master, University of Tromso, $56 \mathrm{p}$.

Frantsvåg J. E., (2011b), The Mega-Journals Are Coming!, in Sciecom info, vol. 7, nº 3.

GFII, (2009), L'édition scientifique française en sciences sociales et humaines, rapport, Paris, Groupement Français de l'Industrie de l'Information, $42 \mathrm{p}$.

GFII, (2010), Synthèse des discussions du groupe de travail sur le libre accès, rapport, Paris, Groupement Français de l'Industrie de l'Information, $50 \mathrm{p}$.

Houghton J., (2009), Open Access - What are the economic benefits? A comparison of the United Kingdom, Netherlands and Denmark, rapport, Copenhagen, Knowledge Exchange, 26 p. 
Laakso M. et al., (2011), The Development of Open Access Journal Publishing from 1993 to 2009, in PLoS One, vol. 6, n6, p. e20961+.

Mabe M., (2003), The growth and number of journals, in Serials, vol. 16, n² 2, pp. 191-197.

Minon M. et Chartron G., (2005), État des lieux comparatif de l'offre de revues SHS, FranceEspagne-Italie. Étude réalisée pour le Ministère de l'éducation nationale, de l'enseignement supérieur et de la recherche, rapport, Paris, Ministère de l'éducation nationale, de l'enseignement supérieur et de la recherche (MESR), $131 \mathrm{p}$.

Morris S., (2006), When is a journal not a journa ? A closer look at the DOAJ, in Learned Publishing, vol. 19, n' 1, pp. 73-76.

Mounier P., (2010), Le libre accès : entre idéal et nécessité, in Hermès, n 57, pp. 23-30.

Pinfield S., (2010), Paying for open access? Institutional funding streams and OA publication charges, in Learned Publishing, vol. 23, $\mathrm{n}^{\circ}$ 1, pp. 39-52.

Polydoratou P. et al., (2010), Open Access Publishing: An Initial Discussion of Income Sources, Scholarly Journals and Publishers, in Chowdhury G. et al. (dir.), The Role of Digital Libraries in a Time of Global Change, vol. 6102 of Lecture Notes in Computer Science, chap. 30, pp. 250-253. Berlin, Heidelberg, Springer, $270 \mathrm{p}$.

RIN, (2008), Activities, costs and funding flows in the scholarly communications system, rapport, London, Research Information Network, 88 p.

Seadle M., (2011), Archiving in the networked world : by the numbers, in Library Hi Tech, vol. 29, $\mathrm{n}^{\circ} 1$, pp. 189-197.

Suber P., (2011), Open Access in 2010, in SPARC Open Access Newsletter, $\mathrm{n}^{\circ} 153$.

Vajou M. et al., (2009), Les enjeux économiques de l'édition scientifique, technique et médicale : Analyses et questions clés, in Les Cahiers du Numérique, vol. 5, n² 2, pp. 143-172.

Walters W. H. et Linvill A., (2011), Characteristics of Open Access Journals in Six Subject Areas, in College \& Research Libraries, vol. 72, $\mathrm{n}^{\circ}$ 2, pp. 372-392.

Tous les sites consultés entre le 6 juin et le 4 juillet 2011

\section{NOTES}

1. Directory of Open Access Journals de l'université de Lund, http://www.doaj.org/.

2. Stevan Harnad estime que leur part pourrait atteindre $25 \%$ du Web of Science en 2020, cf. « The Three Reasons Gold OA Is Premature », 11 juin 2011, http://openaccess.eprints.org/index.php?/ archives/819-The-Three-Reasons-Gold-OA-Is-Premature.html.

3. Study of Open Access Publishing, cf. http://project-soap.eu/.

4. Cf. par exemple (Bascones Dominguez, 2006), (Dewatripont et al., 2006), (RIN 2008), (Houghton, 2009), (Chartron, 2010), (Pinfield, 2010).

5. L'extraction date du 6 juin 2011.

6. http://ulrichsweb.serialssolutions.com.

7. Date du fichier : $1^{\mathrm{er}}$ juin 2011.

8. Critères d'extraction : caractère scientifique, vivant, avec un comité de lecture, pas de monographie en série, pas de magazine professionnel.

9. Le site de l'éditeur présente 10 revues en libre accès, http://publications.edpsciences.org/. 
10. Springer France, EDP Sciences, GERFLINT Groupe d'Études et de Recherches pour le Français Langue Internationale, John Libbey Eurotext, etc.

11. Pour Ulrichsweb s'ajoute une indexation assez restrictive de la notion de « revue » qui exclut par exemple pour la France plus de 200 périodiques à caractère scientifique (séries de monographies, etc.) et une centaine d'éditeurs. Pour la distribution des éditeurs en fonction de leur taille, cette différence n'a pas d'impact significatif.

12. Elektronische Zeitschriftenbibliothek de l'université de Regensburg, avec 54801 revues numériques, http://rzblx1.uni-regensburg.de/ezeit.

13. Le Open Science Directory contient plus de 13000 titres, cf. http:// www.opensciencedirectory.net/.

14. 291 titres OA en juin 2011, http://www.revues.org/.

15. Nous écartons ici la fonction archivage des anciens numéros prise en charge par un autre acteur public, PERSEE.

16. A cause de la politique de sélection du DOAJ qui ne contient pas de revues mixtes ou avec embargo.

17. Avec une moyenne de $20 \%$ pour l'ensemble des disciplines.

18. Un fait que Frantsvåg (2011a) dénonce à juste titre comme cost invisibility problem.

19. La longévité des petites revues en libre accès a été mise en question par Morris (2006).

20. Cf. par exemple l'analyse réalisée par Duvinage (2011).

21. Directement (modèle du « paiement par l'auteur ») ou indirectement (frais de publication d'une revue $\mathrm{OA})$.

22. Un niveau de concentration par ailleurs souvent critiqué.

23. Le développement d'une offre OA par les éditeurs commerciaux, le rachat de BioMed Central par Springer, etc.

24. Cf. l'étude d'Emma Bester sur les services à valeur ajoutée des archives ouvertes (Bester, 2010).

\section{RÉSUMÉS}

L'article analyse la distribution des éditeurs de revues en libre accès en fonction de leur taille. L'analyse exploite l'information sur les revues en libre accès du répertoire DOAJ et l'information sur l'édition des revues scientifiques du Ulrichsweb. Les éditeurs et revues en libre accès sont moins nombreux que ceux du modèle traditionnel mais leur nombre augmente plus rapidement. Par rapport à l'édition traditionnelle, l'édition en libre accès est moins concentrée, avec moins de grands éditeurs et moins de revues publiées par de grands éditeurs. La situation "fragmentée » désavantage l'édition en libre accès du point de vue économique. La mutualisation de la production éditoriale est une des pistes d'évolution. La commercialisation liée à une concentration accrue en est une autre.

The paper examines the distribution of open access (OA) journal publishers in terms of their reach. The analysis uses information on open access journals from the DOAJ Directory as well as information on scientific journals from the Ulrichsweb. Although there are fewer OA journals and publishers than for the traditional model, their number is growing faster. Compared to traditional publishing, OA publishing is less concentrated, with fewer major publishers and fewer journals published by major publishers. Economically speaking, this fragmentation 
disadvantages OA publishing. The sharing of editorial production is one of several possible developments. Commercialization with increased concentration is another.

INDEX

Keywords : academic journals, open access, scientific publishers, economic analysis, efficiency Mots-clés : revues scientifiques, libre accès, éditeurs scientifiques, analyse économique, efficience

\section{AUTEURS}

JOACHIM SCHOPFEL

UDL3, GERIICO - Université Lille 3

\section{HÉLÈNE PROST}

Institut de l'Information Scientifique et Technique (INIST-CNRS) - 54-Vandoeuvre-lès-Nancy

JAN ERIK FRANTSVÅG

Université de Tromsø - Norvège 